Research Article

\title{
Development of Array-Based Gold Nanoclusters for Discrimination of CA125 Overexpressed Serum Samples
}

\author{
Yi Shi, ${ }^{1,2}$ Xianhui Gao, ${ }^{3}$ Wei Wei $\left(\mathbb{D},{ }^{3}\right.$ and Youguo Chen ${ }^{1}{ }^{1}$ \\ ${ }^{1}$ Department of Obstetrics and Gynecology, The First Affiliated Hospital of Soochow University, Suzhou 215006, Jiangsu, China \\ ${ }^{2}$ Department of Obstetrics and Gynecology, The First Affiliated Hospital of Jinzhou Medical University, \\ Jinzhou 121001, Liaoning, China \\ ${ }^{3}$ Department of Basic Science, Jinzhou Medical University, Jinzhou, China
}

Correspondence should be addressed to Wei Wei; 972302574@qq.com and Youguo Chen; youguo_chen@163.com

Received 4 June 2021; Revised 16 September 2021; Accepted 27 October 2021; Published 10 November 2021

Academic Editor: Francesco Ruffino

Copyright (C) 2021 Yi Shi et al. This is an open access article distributed under the Creative Commons Attribution License, which permits unrestricted use, distribution, and reproduction in any medium, provided the original work is properly cited.

\begin{abstract}
Here, we reported a simple method for recognizing CA125 overexpressed serum samples using array-based fluorescent gold nanoclusters (AuNCs). The sensing array was fabricated by the combination of various CA125 aptamer functionalized AuNCs. The fluorescence of different aptamer-capped AuNCs was quenched to a different extent in the presence of CA125. By comprehensive analysis of these fluorescence changes, unique patterns were formed when CA125 was overexpressed in serum samples. This strategy is successfully used for discriminating CA125 overexpressed human serum samples, which have great importance for the diagnosis of ovarian cancer in the early stage.
\end{abstract}

\section{Introduction}

Ovarian cancer has shown a serious threat to women's health, accounting for ca. $5 \%$ of all cancer deaths in females [1]. Early diagnosis of ovarian cancer has great importance for saving lives. For instance, the detection of tumor markers can predict ovarian cancer in the early stages [2]. Among the tumor markers, CA125 has been used by the Food and Drug Administration (FDA) as a clinical tumor marker to predict ovarian cancer. The normal blood level of CA125 in a healthy subject is usually less than $35 \mathrm{U} / \mathrm{mL}$, but CA125 is overexpressed in the blood of most ovarian cancer patients. Various methods have been used for the detection of CA125, such as ELISA (enzyme-linked immunosorbent assay), chemiluminescent sensors, piezoelectric sensors, and electrochemical sensors [3-5]. Most of these detection methods require complicated procedures to prepare the sensors [6]. Biosensors have attracted great attention for the detection of CA125 because they have the potential to be developed as point-of-care devices [7]. For instance, Nunna et al. attached gold nanoparticles on interdigitated electrodes as a biosensor for electrochemical detection CA125 within the concentrations of 3500-84000 U/mL[8]. Hamd-Ghadareh et al. developed blue fluorescent carbon dots (CDs) functionalized with CA125 aptamer, and green fluorescent PAMAM-dendrimers/AuNPs were used at the same time. These two fluorescence sources with different colors were fabricated for generating fluorescence resonance energy transfer (FRET) phenomena between CDs and AuNPs. Such FRET sensor achieved a much more sensitive measurement $(0.5 \mathrm{fg} / \mathrm{mL})$ of CA 125 with the range $1.0 \mathrm{pg} / \mathrm{mL}$ to $1.0 \mathrm{ng} / \mathrm{mL}$ [9]. However, the repeatability of a relatively complicated method may be a concern. The exploration of more repeatable and reliable techniques to detect the abnormal expression of CA125 in the blood is still in requirement.

AuNCs have been used as green fluorescent sensors showing advantages such as low toxicity and easy preparation $[10,11]$. Despite their potential for analysis 
applications such as metal ions, organic drugs, and environmental pollutants [12-15], they have been rarely used for the detection of tumor markers. This is possible because researchers mainly focus on the development of specific sensors. Normally, a specific sensor relies on a specific bioreceptor to specifically analyze one analyte at one time. In addition, the specific detection of a tumor maker by AuNCs requires complicated functionalization processes [16]. On the other hand, array-based sensors, which are also called "chemical nose" methods [17], have been used for the detection of cancers [18]. A sensor array consists of combined sensing elements, whose interactions with various analytes can generate unique patterns, which can discriminate analytes by linear discriminant analysis (LDA), i.e., a method used in statistics to find a linear combination of features that separates different classes of objects. AuNCs have been used for constructing array-based sensors utilizing charge transfer-induced fluorescence changes. However, AuNCbased arrays have never been used for differentiation of tumor markers either possibly because the charge transfer system cannot show significant responses in such cases [19].

Aptamers can specifically interact with the tumor markers and induce remarkable responses. However, aptamers attached closely to fluorophores are hardly applied for discrimination of certain types of samples because the fluorescence is difficult to be changed [20]. Herein, various aptamers were mixed with AuNCs, and the array-based sensors were constructed directly for differentiating samples with different CA125 levels. Aptamers initially interacted with AuNCs loosely and induced their morphology change due to the soft aggregations. Various AuNC-based systems provide different fluorescent responses such as response 1, response 2, and response 3 (Figure 1). Initially, the aptamermodified AuNCs could be placed on a sensing pad and show the fluorescence signal changes depending on the interaction force between the aptamer and AuNCs. In the presence of CA125, AuNCs were competed to CA125 to interact with these aptamers. Once CA125 has a stronger interaction force with the aptamers, the fluorescence changes again. These different changes enable the formation of different factors such as factor 1 , factor 2 , and factor 3 with different patterns. That is to say, AuNCs can finally have different fluorescence intensities after the interaction with the same concentration of CA 125 because the intermediate aptamer is different. Next, LDA can differentiate the CA125 factors in a liquid such as the overexpressed serum samples. To the best of our knowledge, this is the first report for constructing AuNCs as array-based sensors for the detection of tumor markers.

\section{Experiments}

Transmission electron microscopy (TEM) was performed by using a JEOL JEM-2100 microscope operating at $200 \mathrm{kV}$. The fluorescence was obtained using a microreader (Varioskan LUX). Serum samples of CA125 with different levels were obtained from the first afflicted hospital of Jinzhou Medical University. CA125 was clinically detected by the chemiluminescent system (ARCHITECT i4000). The aptamers used were designed with the modification according to references $[21,22]$ which are listed in Table 1 and were obtained from Sangon Biotech (Shanghai) Co., Ltd. All other reagents were obtained from Aladdin company, which were of analytical grades. Deionized water was used through the experiments.

For the synthesis of AuNCs, $100 \mu \mathrm{L}$ of $150 \mathrm{mM}$ glutathione (GSH) and $\mathrm{HAuCl}_{4}(20 \mathrm{mM}, 0.50 \mathrm{~mL})$ were mixed with $4.35 \mathrm{~mL}$ of deionized water at room temperature. The mixture was transferred to a water bath and heated at $70^{\circ} \mathrm{C}$ for $24 \mathrm{~h}$. The array-based sensors were obtained by adding $10 \mu \mathrm{L}$ of $(2 \mathrm{mM})$ AuNCs into the PBS solutions (10 mM, pH 7.4). Various CA125 aptamers $(10 \mu \mathrm{L}, 100 \mathrm{nM})$, as shown in Table 1, were added into different AuNCs solutions, respectively. The mixtures were carried for 1 hour. Then, serum samples of CA125 with different levels were combined with the above solutions, respectively, and the reaction was allowed to process at room temperatures for 1 hour. The fluorescence intensity was measured, and LDA was used to differentiate these samples.

\section{Results and Discussion}

TEM was used to confirm the formation of AuNCs (Figure 2). It can be seen that the clusters were homogeneously distributed (Figure 2(a)), and the average size (Figure 2(b)) was around $1.4 \mathrm{~nm}$. This indicates that the ultrasmall AuNCs were successfully fabricated.

TEM (Figure 3) was further used to check AuNCs in the absence and presence of the aptamers and CA125. Compared with Figure 2 that revealed the monodispersity of AuNCs in the absence of the aptamers, the aptamer functionalized AuNCs, namely, AuNCs-QS1 (NC1), AuNCs-Q1 (NC2), AuNCs-QN1 (NC3), AuNCs-PS1 (NC4), AuNCs-P1 (NC5), and AuNCs-PN1 (NC6) suffered different aggregations, but most of these changes were insignificant. These aggregations are different from the fused aggregations that result in the formation of large particles. Furthermore, in the presence of CA125, the aptamer functionalized AuNCs all show certain degrees of aggregations or disaggregations, resulting in the quenching or enhancement of the fluorescence to different degrees. By analyzing these comprehensive changes, the system is reliable to reflect the presence of CA125.

For the differentiability analysis, 3 types of artificial bovine serum samples (CA125>35 u/mL, Ca125 closed to $35 \mathrm{u} / \mathrm{mL}$, and CA $125<35 \mathrm{u} / \mathrm{mL}$ ) were chosen as sensing targets. As illustrated in Figure 4(a), the bovine serum samples with different levels of CA125 resulted in unique fluorescence response patterns due to different interactions with the aptamers, which induce the corresponding morphology change of AuNCs. The fluorescence intensity patterns were subjected to LDA (Figure 4(b)), and different CA125 level samples were well separated. This indicates that the current system can differentiate CA125 overexpressed samples $(>35 \mathrm{U} / \mathrm{mL})$ from healthy subjects. 

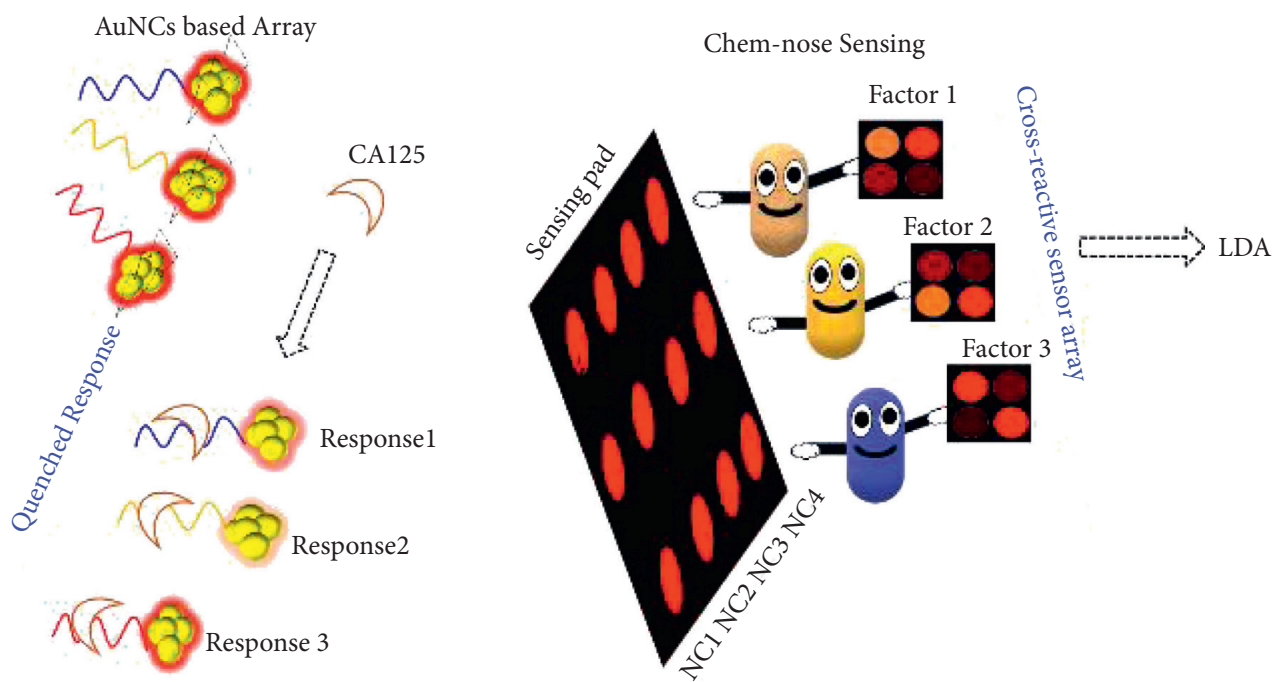

Figure 1: Quenching of the aptamer functionalized AuNCs to different extents and the array-based sensing system for the detection of CA125.

TABLE 1: Aptamers for constructing the array-based sensing system.

\begin{tabular}{|c|c|}
\hline Aptamer & Sequence \\
\hline QS1 & 5'-TAATACGA CTCACTATAGGGAGACAAGAATAAACGCTCAA-3' \\
\hline Q1 & 5'-SH-(CH2)6TAATACGA CTCACTATAGGGAGACAAGAATAAACGCTCAA-3' \\
\hline QN1 & 5'-NH2-(CH2)6TAATACGA CTCACTATAGGGAGACAAGAATAAACG-3' \\
\hline PS1 & $5^{\prime}$-ACT TCA GTGAGT TGT CCC ACG GTC GGC GAG TCG GTG GTAG-3' \\
\hline $\mathrm{P} 1$ & $5^{\prime}$-SH-(CH2)6-ACT TCA GTGAGT TGT CCC ACG GTC GGC GAG TCG GTG GTAG-3' \\
\hline PN1 & $5^{\prime}$-NH2-(CH2)6-ACT TCA GTGAGT TGT CCC ACG GTC GGC GAG TCG GTG GTAG-3' \\
\hline
\end{tabular}

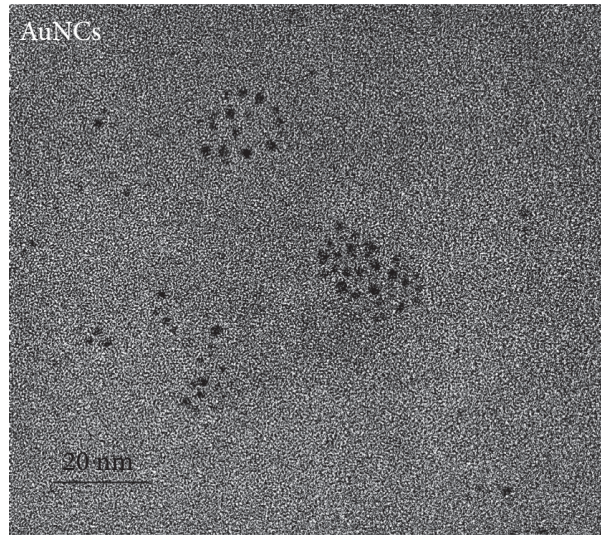

(a)

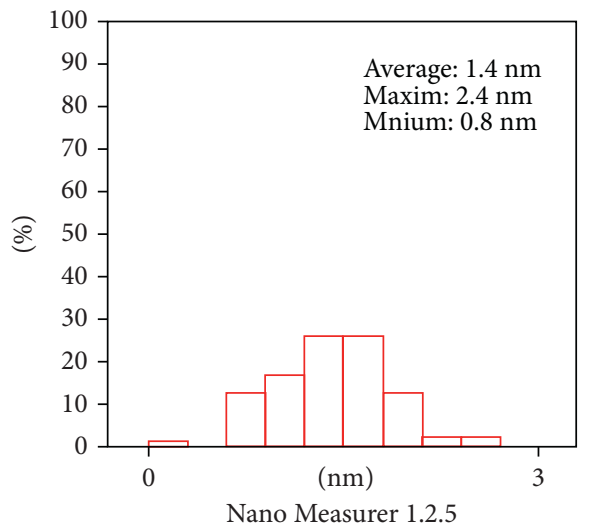

(b)

Figure 2: TEM of the original GSH-protected AuNCs.

We tested blinded samples with the AuNC-based sensor arrays. Six unknown human serum samples were randomly selected from the three groups (high, medium, and low levels of CA125) and analyzed by the sensor array (Figure 5) and clinically chemiluminescent methods. As shown in Figure 5(a), different fluorescent responses occurred when the human serum samples of three groups were combined with the sensor array. LDA revealed that serums of CA125 

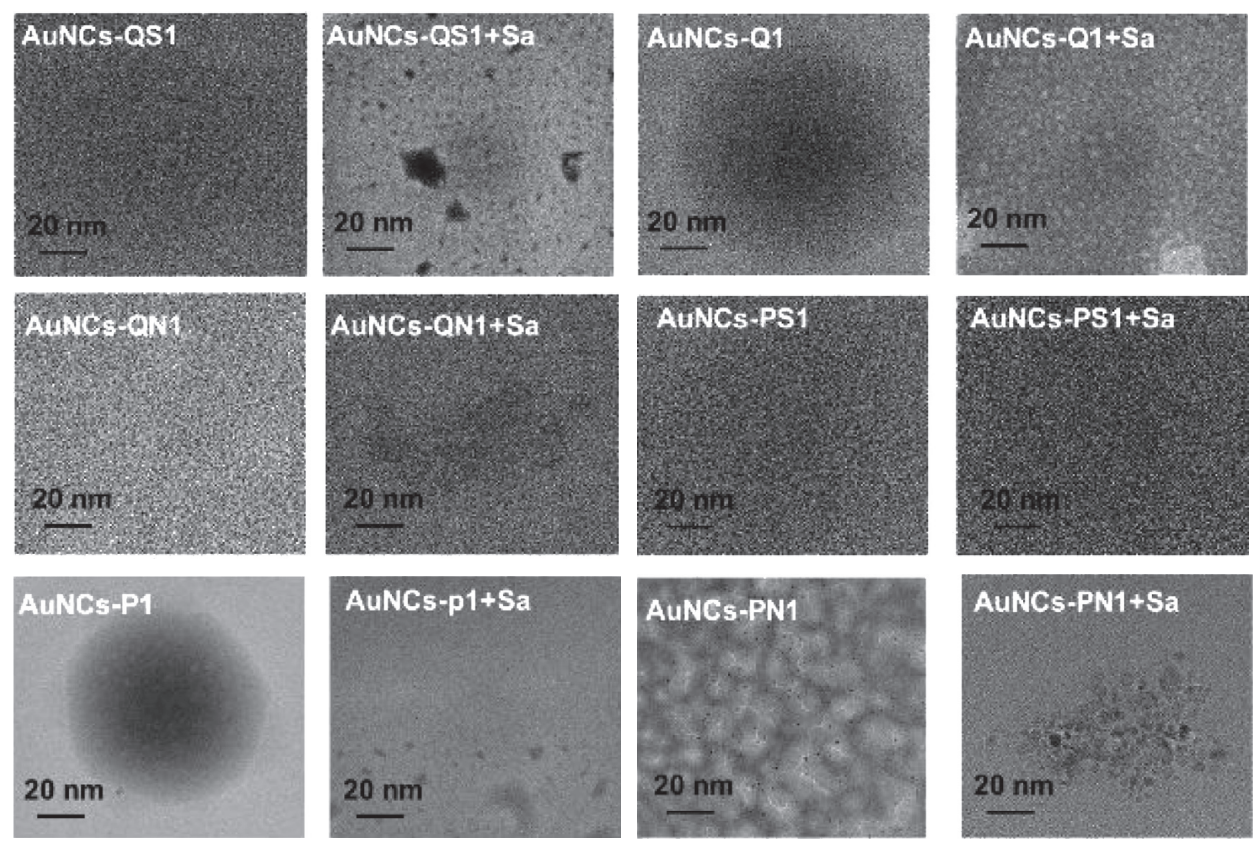

Figure 3: TEM of AuNCs in the presence of the aptamers and CA125.

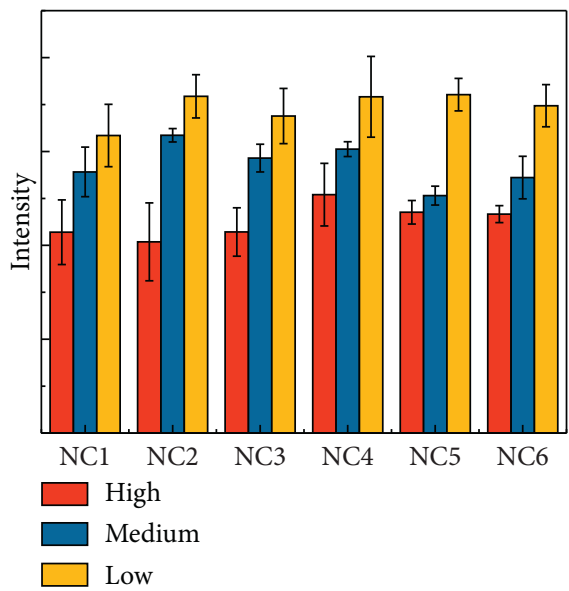

(a)

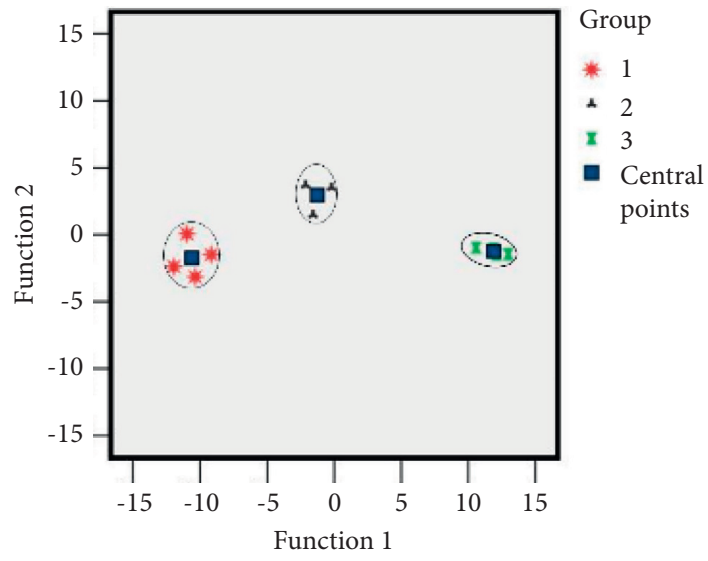

(b)

Figure 4: Array-based sensing of artificial bovine serum samples with different CA125 levels. (a) Fluorescence emission intensity (610 nm) patterns of the 3 types of samples (low, medium, and high level of CA125) on the AuNCs array as an average of 4 parallel measurements. (b) LDA shows the excellent separation of the samples with different levels of CA125.

overexpressed samples could be discriminated from those samples with normal CA125 levels (Figure 5(b)). According to the clinically standard results (Table 2), all six serum samples were correctly identified, corresponding to an identification accuracy of $100 \%$. Overall, it could be concluded that the developed sensor array is promising for recognizing CA125 overexpressed serum samples. By comparing with traditional chemiluminometric methods, which were verified by the clinical diagnosis, the results also showed high accuracy and repeatability.
This array sensor can also be used as a specific sensor like the traditional method by selecting one of the aptamermodified AuNCs. Figure 6 shows the fluorescence spectra (Figure 6(a)) of aptamer 1 (QS1) modified AuNCs, and the corresponding fluorescence change (Figure 6(b)) could be used as the calibration graph for linear detection of CA125 antigen. Similarly, the other aptamer-modified AuNCs all have their calibration graphs. Herein, we combined with aptamer-modified AuNCs for the group-sensing of the analytes. Multiple sensor elements were used for the 


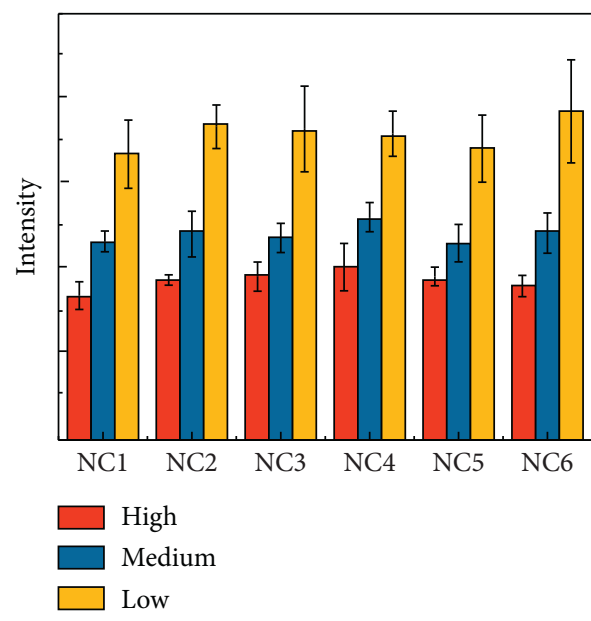

(a)
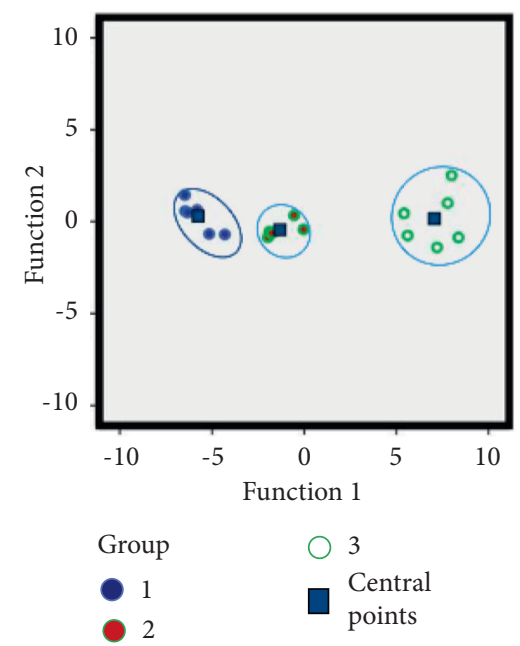

(b)

FIGURE 5: Array-based sensing of human serum samples with different CA125 levels. (a) Fluorescence emission intensity (610 nm) patterns of the 3 types of samples (low, medium, and high level of CA125) on the AuNCs array as an average of six patient samples. (b) LDA shows the excellent separation of the samples with different levels of CA125.

TABLE 2: Determination of CA125 by the traditional chemiluminescence method.

\begin{tabular}{lccc}
\hline Test & High & Medium & Low \\
\hline 1 & $>1000$ & 29.7 & $<20$ \\
2 & 151.4 & 28.9 & $<20$ \\
3 & 294.9 & 30.8 & $<20$ \\
4 & 355.4 & 29.7 & $<20$ \\
5 & $>1000$ & 24.9 & $<20$ \\
6 & 882.6 & 29.1 & $<20$ \\
\hline
\end{tabular}

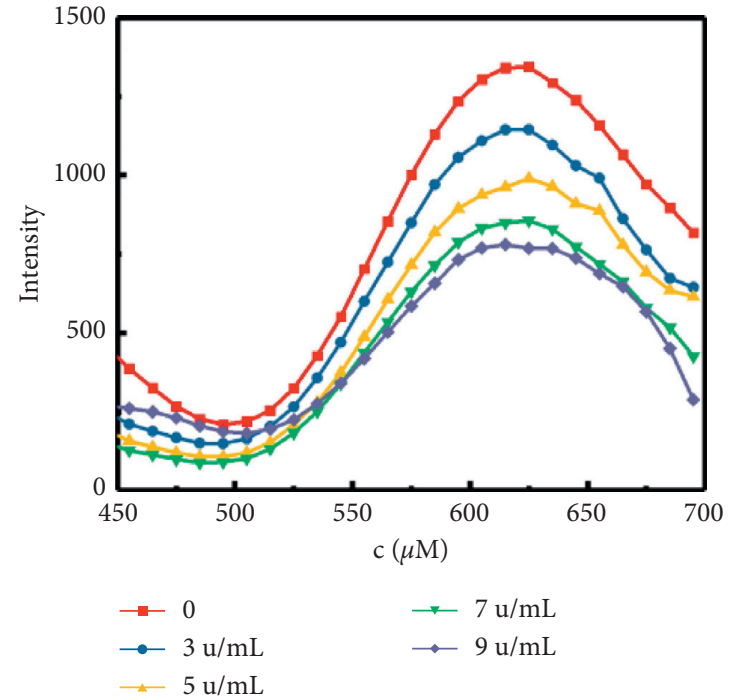

(a)

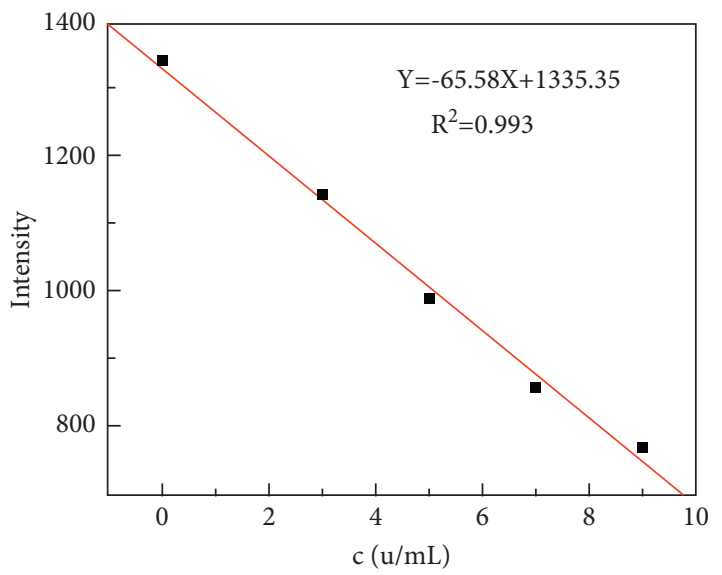

(b)

FIGURE 6: Fluorescence spectra (a) and normalized fluorescence intensity at $610 \mathrm{~nm}$ (b) with the increases in concentrations of CA125 from 0 to $9 \mathrm{u} / \mathrm{mL}$; the excitation wavelength was $365 \mathrm{~nm}$ 
detection of CA125 at the same time, which could provide more accurate data. Because of the repeated verification of multiple factors, this sensor array will have unique reproducibility. Such a design will also provide a robust strategy for various other biomarkers.

\section{Conclusions}

In this study, we developed a sensor array using six kinds of fluorescent AuNCs with the corresponding aptamers as sensing receptors for CA125 discrimination. Different levels of CA125 expressed samples can be distinguished from each other in human serum samples. This work opens a path for constructing sensor arrays for the diagnosis of tumor markers in clinical practice.

\section{Data Availability}

The data generated or analyzed during this study are included in this article.

\section{Conflicts of Interest}

The authors declare that they have no conflicts of interest.

\section{Acknowledgments}

The authors acknowledge Jinzhou Medical University and the affiliated hospital for the instrument, clinical data, and financial support.

\section{References}

[1] Z. Momenimovahed, A. Tiznobaik, S. Taheri, and H. Salehiniya, "Ovarian cancer in the world: epidemiology and risk factors," International Journal of Women's Health, vol. 11, pp. 287-299, 2019.

[2] H. J. Whitwell, J. Worthington, O. Blyuss et al., "Improved early detection of ovarian cancer using longitudinal multimarker models," British Journal of Cancer, vol. 122, no. 6, pp. 847-856, 2020.

[3] A. Sangili, T. Kalyani, S.-M. Chen, A. Nanda, and S. K. Jana, "Label-Free electrochemical immunosensor based on onestep electrochemical deposition of AuNP-rgo nanocomposites for detection of endometriosis marker CA 125," ACS Applied Bio Materials, vol. 3, no. 11, pp. 7620-7630, 2020.

[4] A. Saadati, S. Hassanpour, F. Bahavarnia, and M. Hasanzadeh, "A novel biosensor for the monitoring of ovarian cancer tumor protein CA 125 in untreated human plasma samples using a novel nano-ink: a new platform for efficient diagnosis of cancer using paper based microfluidic technology," Analytical Methods, vol. 12, no. 12, pp. 1639-1649, 2020.

[5] B. Fatima, D. Hussain, S. Bashir et al., "Catalase immobilized antimonene quantum dots used as an electrochemical biosensor for quantitative determination of $\mathrm{H} 2 \mathrm{O} 2$ from CA-125 diagnosed ovarian cancer samples," Materials science \& engineering. C, Materials for biological applications, vol. 117, Article ID 111296, 2020.

[6] F. Chen, Y. Liu, C. Chen, H. Gong, C. Cai, and X. Chen, "Respective and simultaneous detection tumor markers CA125 and STIP1 using aptamer-based fluorescent and RLS sensors," Sensors and Actuators B: Chemical, vol. 245, pp. 470-476, 2017.

[7] N. Razmi and M. Hasanzadeh, "Current advancement on diagnosis of ovarian cancer using biosensing of CA 125 biomarker: analytical approaches," TRAC Trends in Analytical Chemistry, vol. 108, pp. 1-12, 2018.

[8] B. B. Nunna, D. Mandal, J. U. Lee et al., "Detection of cancer antigens (CA-125) using gold nano particles on interdigitated electrode-based microfluidic biosensor," Nano Convergence, vol. 6 , no. 1, p. 3, 2019

[9] S. Hamd-Ghadareh, A. Salimi, F. Fathi, and S. Bahrami, "An amplified comparative fluorescence resonance energy transfer immunosensing of CA125 tumor marker and ovarian cancer cells using green and economic carbon dots for bio-applications in labeling, imaging and sensing," Biosensors and Bioelectronics, vol. 96, pp. 308-316, 2017.

[10] C. N. Loynachan, A. P. Soleimany, J. S. Dudani et al., "Renal clearable catalytic gold nanoclusters for in vivo disease monitoring," Nature Nanotechnology, vol. 14, no. 9, pp. 883-890, 2019.

[11] J. Zhang, C. Cai, S. Razzaque, I. Hussain, Q.-W. Lu, and B. Tan, "Synthesis of water-soluble and highly fluorescent gold nanoclusters for $\mathrm{Fe} 3+$ sensing in living cells using fluorescence imaging," Journal of Materials Chemistry B, vol. 5, no. 28, pp. 5608-5615, 2017.

[12] M. L. Desai, H. Basu, S. Saha, R. K. Singhal, and S. K. Kailasa, "Fluorescence enhancement of bovine serum albumin gold nanoclusters from $\mathrm{La} 3+$ ion: detection of four divalent metal ions ( $\mathrm{Hg} 2+, \mathrm{Cu} 2+, \mathrm{Pb} 2+$ and $\mathrm{Cd} 2+)$," Journal of Molecular Liquids, vol. 336, 2021.

[13] S. Akavaram, M. L. Desai, T.-J. Park, Z. V. P. Murthy, and S. K. Kailasa, "Trypsin encapsulated gold-silver bimetallic nanoclusters for recognition of quinalphos via fluorescence quenching and of $\mathrm{Zn} 2+$ and $\mathrm{Cd} 2+$ ions via fluorescence enhancement," Journal of Molecular Liquids, vol. 327, 2021.

[14] S. K. Kailasa, M. R. Kateshiya, and N. I. Malek, "Introduction of cellulose-cysteine Schiff base as a new ligand for the fabrication of blue fluorescent gold nanoclusters for the detection of indapamide drug," Journal of Molecular Liquids, vol. 319, 2020.

[15] J. R. Bhamore, S. Jha, R. K. Singhal, Z. V. P. Murthy, and S. K. Kailasa, "Amylase protected gold nanoclusters as chemoand bio- sensor for nanomolar detection of deltamethrin and glutathione," Sensors and Actuators B: Chemical, vol. 281, pp. 812-820, 2019.

[16] D. Li, G. Wang, and X. Mei, "Diagnosis of cancer at early stages based on the multiplex detection of tumor markers using metal nanoclusters," Analyst, vol. 145, no. 22, pp. 7150-7161, 2020.

[17] Y. Geng, W. J. Peveler, and V. M. Rotello, "Array-based "chemical nose" sensing in diagnostics and drug discovery," Angewandte Chemie International Edition, vol. 58, no. 16, pp. 5190-5200, 2019.

[18] L. Digiacomo, K. Jafari-Khouzani, S. Palchetti et al., "A protein corona sensor array detects breast and prostate cancers," Nanoscale, vol. 12, no. 32, pp. 16697-16704, 2020.

[19] S. Xu, X. Lu, C. Yao et al., "A visual sensor array for pattern recognition analysis of proteins using novel blue-emitting fluorescent gold nanoclusters," Analytical Chemistry, vol. 86, no. 23, pp. 11634-11639, 2014.

[20] V. Gedi, C. K. Song, G. B. Kim et al., "Sensitive on-chip detection of cancer antigen 125 using a DNA aptamer/carbon nanotube network platform," Sensors and Actuators B: Chemical, vol. 256, pp. 89-97, 2018. 
[21] H. Jin, R. Gui, J. Gong, and W. Huang, "Aptamer and 5fluorouracil dual-loading $\mathrm{Ag} 2 \mathrm{~S}$ quantum dots used as a sensitive label-free probe for near-infrared photoluminescence turn-on detection of CA125 antigen," Biosensors and Bioelectronics, vol. 92, pp. 378-384, 2017.

[22] M. Negahdary, "Aptamers in nanostructure-based electrochemical biosensors for cardiac biomarkers and cancer biomarkers: a review," Biosensors and Bioelectronics, vol. 152, Article ID 112018, 2020. 